\title{
Can a wormhole generate electromagnetic field?
}

\author{
Mubasher Jamil ${ }^{1}$ \\ ${ }^{1}$ Center for Advanced Mathematics and Physics, \\ National University of Sciences and Technology, \\ Rawalpindi, 46000, Pakistan
}

\begin{abstract}
We have considered the possibility of a rotating wormhole surrounded by a cloud of charged particles. Due to slow rotation of the wormhole, the charged particles are dragged, thereby producing an electromagnetic field. We have determined the strength of this electromagnetic field and the corresponding flux of radiation.
\end{abstract}




\section{INTRODUCTION}

Traversable wormhole arises as a solution to the Einstein field equations and was first proposed by Morris and Thorne [1, 2] as time travel machines. The idea of wormhole spacetime was given by J.A. Wheeler in his attempt to apply quantum mechanics at the Planck scale. The resulting spacetime turns out to be fluctuating giving rise a number of topologies including the wormhole [3]. A static and spherically symmetric wormhole possesses interesting geometry having a throat that flares out in two opposite directions. The throat connects either two different asymptotically flat regions in the same spacetime or entirely two distinct spacetimes. Later on, Ellis [4] termed this geometry as a 'drainhole' that could render particle motion from either mouths. The throat has the tendency to get closed in a very short time (of the order of Planck time) thereby limiting the time travel possibility. In order to create a stable wormhole, a negative energy (or the exotic matter) is required to keep the wormhole's throat open. Such a negative energy thus violates the null energy condition (NEC) i.e. $T_{\mu \nu} u^{\mu} u^{\nu} \geq 0$, where $T_{\mu \nu}$ is the stress energy tensor and $u^{\mu}$ is the future directed null vector. Since NEC is the weakest energy condition, it implies that all the energy conditions (weak, strong and dominant) will be violated automatically. These energy conditions are generally obeyed by the classical matter but are violated by certain quantum fields which exhibit the Casimir effect and the Hawking evaporation process [5].

In the context of cosmology, the phantom like dark energy with the equation of state (EoS) $\omega<-1$ violates the NEC and has been analyzed as a source to sustain traversable wormholes [6, 7]. It has been shown that arbitrary small amount of phantom energy can support the existence of wormhole [8 11]. It is argued in [12] that traversable wormhole can be constructed from the black hole by absorbing exotic energy and conversely the wormhole can collapse to a black hole by releasing exotic energy. Surprisingly, the wormhole can lead to inflationary universe by absorbing arbitrarily large amount of exotic matter. Moreover, the size of the wormhole can be increased or decreased by a corresponding increase or decrease of the absorption of exotic matter [13].

Earlier, it has been shown that neutral test particles propagating towards the rotating wormhole radially, start moving about the wormhole in the spiral path. After entering the wormhole's throat, the particles pass through the throat and move away from the throat following the spiral trajectories [14]. We are here interested in a stationary and axially sym- 
metric wormhole having non-zero angular velocity surrounded by the continuum of charged particles that are dragged by the wormhole in the angular direction. The rotation is assumed to be negligible so that quadratic terms in the angular velocity are ignored. The frame dragging effects on the charged particles produces a poloidal electromagnetic field. The resulting field around the wormhole is determined under the slow rotation approximation. This feature of the wormhole physics can be of interest from astronomical point of

view. It has been suggested that wormholes can cause gravitational lensing events [15]. Also the accretion of matter on a wormhole could also create a black hole [16]. In this connection, the electromagnetic structure of rotating wormholes is investigated here.

The outline of this paper is as follows: In the next section, we formulate the governing equations of our gravitational system and then solve them in the third section. Finally, we conclude in the fourth section.

\section{FORMULATION OF THE DYNAMICAL SYSTEM}

We consider a stationary and axially symmetric wormhole, surrounded by the charged particles, is given by (in geometrical units $G=1=c$ ) [5]:

$$
d s^{2}=-N^{2} d t^{2}+e^{\mu} d r^{2}+r^{2} K^{2}\left[d \theta^{2}+\sin ^{2} \theta(d \phi-\omega d t)^{2}\right],
$$

where

$$
e^{-\mu(r, \theta)}=1-\frac{b(r, \theta)}{r},
$$

with $N, K, \mu$ and $\omega$ are functions of $r$ and $\theta$ only. The function $b(r, \theta)$ determines the spatial shape of the wormhole and is thus called the shape function. Also $\omega(r, \theta)$ is the orbital angular velocity of the wormhole. More precisely

$$
\omega \equiv \frac{d \phi}{d t}=\frac{d \phi / d \tau}{d t / d \tau}=\frac{u^{\phi}}{u^{t}}
$$

where $u^{\phi}$ and $u^{t}$ are azimuthal and transverse components of the four velocity respectively. Notice that static and spherically symmetric Morris-Thorne wormhole [1] is obtained from Eq. (1) by doing the following substitution

$$
N(r, \theta)=e^{2 \Phi(r)}, \quad b(r, \theta)=b(r), \quad K(r, \theta)=1, \quad \omega(r, \theta)=0 .
$$


Above the redshift function $\Phi(r)$ determines the gravitational redshift. In the slow rotation approximation $O\left(\omega^{2}\right)$, Eq. (1) gives

$$
d s^{2} \approx-N^{2} d t^{2}+e^{\mu} d r^{2}+r^{2} K^{2}\left[d \theta^{2}+\sin ^{2} \theta\left(d \phi^{2}-2 \omega d t d \phi\right)\right]
$$

The determinant of the metric Eq. (5) is

$$
\left|g_{\alpha \beta}\right|=g=-e^{\mu} r^{4} K^{4} N^{2} \sin ^{2} \theta
$$

where we have neglected terms in $O\left(\omega^{2}\right)$. The relativistic Maxwell equations are

$$
\begin{gathered}
F_{[\alpha \beta, \gamma]}=0, \\
\left(\sqrt{-g} F^{\alpha \beta}\right)_{, \beta}=4 \pi J^{\alpha} .
\end{gathered}
$$

Above $F_{\alpha \beta}$ is the electromagnetic field tensor and $J^{\alpha}$ is the four vector current density of the charged particles. The former is given by [17]

$$
F_{\alpha \beta}=u_{\alpha} E_{\beta}-u_{\beta} E_{\alpha}+\eta_{\alpha \beta \gamma \delta} u^{\gamma} B^{\delta}
$$

Here $u^{\alpha}$ is the four velocity of the charged particles producing an electromagnetic field having $E^{\alpha}$ and $B^{\alpha}$ as four vector electric and magnetic field components. The current density containing the convection and the conduction currents is given by [17]

$$
J^{\alpha}=\epsilon u^{\alpha}+\sigma u_{\beta} F^{\beta \alpha}
$$

Here $\epsilon$ and $\sigma$ are the charge density and the fluid conductivity respectively. Moreover, the volume four element is given by [18]

$$
\eta_{\alpha \beta \gamma \delta}=\sqrt{-g} \epsilon_{\alpha \beta \gamma \delta}, \quad \eta^{\alpha \beta \gamma \delta}=-\frac{1}{\sqrt{-g}} \epsilon^{\alpha \beta \gamma \delta} .
$$

Above $\epsilon_{\alpha \beta \gamma \delta}$ is the Levi-Civita symbol. We now consider a zero angular momentum observer (ZAMO) moving about the slowly rotating wormhole in the equatorial plane $(\theta=\pi / 2)$ at a fixed distance from the wormhole's axis of rotation $(r=R)$ which yield $u^{r}=0=u^{\theta}$. The four velocity of ZAMO is given by

$$
u^{\alpha}=N^{-1}(r, \theta)(1,0,0, \omega(r, \theta)), u_{\alpha}=N(r, \theta)(-1,0,0,0),
$$

which satisfy the normalization condition $u^{\alpha} u_{\alpha}=-1$. Thus the electromagnetic field around the wormhole is determined from Eqs. (7) and (8).

Assuming the neutrality of plasma $\left(J^{\alpha}=0\right)$ and the electromagnetic field to be function of $r$ and $\theta$ only, equations (7) for the metric in Eq. (5) become 


$$
\begin{aligned}
\left(r^{2} e^{\mu / 2} K^{2} \sin \theta B^{r}\right)_{, r}+\left(r^{2} e^{\mu / 2} K^{2} \sin \theta B^{\theta}\right)_{, \theta} & =0, \\
\left(N E_{\phi}\right)_{, r} & =0, \\
\left(N E_{\phi}\right)_{, \theta} & =0, \\
\left(N E_{\theta}+\omega r^{2} e^{\mu / 2} K^{2} \sin \theta B^{r}\right)_{, r}-\left(N E_{r}+\omega r^{2} e^{\mu / 2} K^{2} \sin \theta B^{\theta}\right)_{, \theta} & =0,
\end{aligned}
$$

while Eq. (8) yields

$$
\begin{aligned}
\left(r^{2} e^{\mu / 2} K^{2} \sin \theta E^{r}\right)_{, r}+\left(r^{2} e^{\mu / 2} K^{2} \sin \theta E^{\theta}\right)_{, \theta} & =0, \\
\left(N B_{\phi}\right)_{, r} & =0, \\
\left(N B_{\phi}\right)_{, \theta} & =0, \\
\left(N B_{\theta}+\omega r^{2} e^{\mu / 2} K^{2} \sin \theta E^{r}\right)_{, r}-\left(N B_{r}+\omega r^{2} e^{\mu / 2} K^{2} \sin \theta E^{\theta}\right)_{, \theta} & =0 .
\end{aligned}
$$

\section{SOLUTION OF GOVERNING EQUATIONS}

To obtain some specific solution of Eqs. (7) and (8), let us assume the following ansatz for the electric and the magnetic fields:

$$
\begin{aligned}
& E_{r}(r, \theta) \equiv k_{1} E_{\theta}(r, \theta) \equiv k_{2} E_{\phi}(r, \theta)=R_{E}(r) \Theta_{E}(\theta), \\
& B_{r}(r, \theta) \equiv k_{3} B_{\theta}(r, \theta) \equiv k_{4} B_{\phi}(r, \theta)=R_{B}(r) \Theta_{B}(\theta) .
\end{aligned}
$$

Here $k_{i}, i=1,2,3,4$ are non-zero dimensional constants. We consider two types of solutions containing either (1) the arbitrary functions $N, K, \mu$ and $\omega$ are functions of $r$ only or (2) the same arbitrary functions are dependent on $\theta$ only.

Case (1). We first assume the arbitrary functions in the Eq. (5) to be dependent on the radial coordinate $r$ only. In the following, the $C_{j}$ where $j=1, . ., 12$ and $D_{l}$ where $l=1, . ., 9$ are constants parameters.

Using Eq. (22) in (13), we get

$$
R_{B}(r)=\frac{D_{1} e^{C_{1} r}}{r^{2} e^{\mu(r) / 2} K^{2}(r)}, \quad \Theta_{B}(\theta)=\frac{D_{2} e^{-C_{1} k_{3} \theta}}{\sin \theta} .
$$


Above $C_{1}$ has dimensions of $L^{-1}$ while $k_{3}$ has dimensions of $L$. Using Eq. (21) in (14) we get

$$
R_{E}(r)=\frac{C_{2}}{N(r)} .
$$

Similarly, using Eq. (21) in (15), we have

$$
\Theta_{E}(\theta)=C_{3}
$$

Finally, using Eqs. (23-25) in (16), we get

$$
\omega(r)_{, r}+2 C_{1} \omega(r)=0
$$

which gives

$$
\omega(r)=D_{3} e^{-2 C_{1} r}
$$

Notice that if $D_{3}$ and $C_{1}$ are positive constants then the angular velocity $\omega$ will decrease as $r$ increases. Making use of Eq. (21) in (17), we get

$$
R_{E}(r)=\frac{D_{4} e^{C_{4} r}}{r^{2} e^{\mu(r) / 2} K^{2}(r)}, \quad \Theta_{E}(\theta)=\frac{D_{5} e^{-C_{4} k_{1} \theta}}{\sin \theta} .
$$

Above $C_{4}$ has dimensions of $L^{-1}$ while $k_{1}$ has dimensions of $L$. Using Eq. (22) in (18) gives

$$
R_{B}(r)=\frac{C_{5}}{N(r)}
$$

Also, Eq. (22) in (19) gives

$$
\Theta_{B}(\theta)=C_{6}
$$

The general solution of the Eqs. (13-20) becomes

$$
\begin{aligned}
& E_{r}(r, \theta)=R_{E}(r) \Theta_{E}(\theta)=\frac{D_{4} D_{5} e^{C_{4}\left(r-k_{1} \theta\right)}}{r^{2} e^{\mu(r) / 2} K^{2}(r) \sin \theta}, \\
& B_{r}(r, \theta)=R_{B}(r) \Theta_{B}(\theta)=\frac{D_{1} D_{2} e^{C_{1}\left(r-k_{3} \theta\right)}}{r^{2} e^{\mu(r) / 2} K^{2}(r) \sin \theta} .
\end{aligned}
$$

Case (2). Let us solve Eqs. (13-20) by choosing the arbitrary functions in Eq. (5) depending on $\theta$ only.

Using Eq. (22) in (13), we get

$$
R_{B}(r)=\frac{D_{5} e^{C_{7} r}}{r^{2}}, \quad \Theta_{B}(\theta)=\frac{D_{6} e^{-k_{3} C_{7} \theta}}{e^{\mu(\theta) / 2} K^{2}(\theta) \sin \theta} .
$$


Above $C_{7}$ has dimensions of $L^{-1}$. Using Eq. (21) in (14), we have

$$
R_{E}(r)=C_{8}
$$

Also using Eq. (21) in (15), we can write

$$
\Theta_{E}(\theta)=\frac{C_{9}}{N(\theta)} .
$$

Using Eqs. (33-35) in (16), we get

$$
\omega(\theta)_{, \theta}-2 C_{7} k_{3} \omega(\theta)=0,
$$

which yields

$$
\omega(\theta)=D_{7} e^{2 C_{7} k_{3} \theta} .
$$

Further using Eq. (21) in (17), we get

$$
R_{E}(r)=\frac{D_{8} e^{C_{10} r}}{r^{2}}, \quad \Theta_{E}(\theta)=\frac{D_{9} e^{-k_{1} C_{10} \theta}}{e^{\mu(\theta) / 2} K^{2}(\theta) \sin \theta} .
$$

Similarly, $C_{10}$ has dimensions of $L^{-1}$. Making use of Eq. (22) in (18), we get

$$
R_{B}(r)=C_{11}
$$

Using Eq. (22) in (19) yields

$$
\Theta_{B}(\theta)=\frac{C_{12}}{N(\theta)} .
$$

From Eqs. (32-38), the components of electric and magnetic field become

$$
\begin{aligned}
& E_{r}(r, \theta)=R_{E}(r) \Theta_{E}(\theta)=\frac{D_{8} D_{9} e^{C_{10}\left(r-k_{1} \theta\right)}}{r^{2} e^{\mu(\theta) / 2} K^{2}(\theta) \sin \theta}, \\
& B_{r}(r, \theta)=R_{B}(r) \Theta_{B}(\theta)=\frac{D_{5} D_{6} e^{C_{7}\left(r-k_{3} \theta\right)}}{r^{2} e^{\mu(\theta) / 2} K^{2}(\theta) \sin \theta} .
\end{aligned}
$$

The Poynting vector $\mathbf{S}=\mathbf{E} \times \mathbf{B}$ determining the flux of radiation from the slowly rotating wormhole is determined to be

$$
\mathbf{S}=E_{\phi} B_{\phi} \Delta \equiv \frac{1}{k_{2} k_{4}} E_{r} B_{r} \Delta \equiv \frac{k_{1} k_{3}}{k_{2} k_{4}} E_{\theta} B_{\theta} \Delta,
$$

where

$$
\Delta \equiv \hat{i}\left(\frac{k_{2}}{k_{1}}-\frac{k_{4}}{k_{3}}\right)-\hat{j}\left(k_{2}-k_{4}\right)+\hat{k}\left(\frac{1}{k_{3}}-\frac{1}{k_{1}}\right) k_{2} k_{4} .
$$




\section{CONCLUSION}

In this paper we have studied the electromagnetic field and the corresponding generation of electromagnetic flux due to a rotating wormhole. The source of this field is the charge density which surrounds the wormhole. The distribution of charges is assumed to be spherically symmetrical. In general, a wormhole spacetime is inhomogeneous and therefore

requires inhomogeneous distribution of matter (or any other source) in its vicinity [19]. But this does not apply here since exterior spacetime of the wormhole contains only electric charges which do not induce any pressure on the wormhole. Also note that metric (1) contains three arbitrary functions, which need to be chosen for any physically motivated model. Our model predicts the production of the electromagnetic field with a certain radiation flux due to wormhole rotation. It is anticipated that this model would explain some bizarre phenomenon processes in the universe like the gamma ray bursts. Finally, the analysis performed in this paper can be extended to charged coupled with rotating wormholes as well [20]. It would also be worth exploring the same procedure for the general class of traversable wormholes solutions derived in [21].

\section{Acknowledgment}

One of us (MJ) would like to thank Farook Rahaman for sharing useful comments to improve this work.

[1] M.S. Morris and K.S. Thorne, Am. J. Phys. 56 (1988) 5.

[2] M.S. Morris et al, Phys. Rev. Lett. 61 (1988) 1446.

[3] J.A. Wheeler, Ann. Phys. 2 (1957) 604.

[4] H.G. Ellis, J. Math. Phys. 14 (1973) 104.

[5] F.S.N. Lobo, gr-qc/0710.4474v1.

[6] F.S.N. Lobo, Phys. Rev. D 71 (2005) 084011.

[7] F.S.N. Lobo, gr-qc/0611150.

[8] F. Rahman et al, gr-qc/0512075v2.

[9] F. Rahaman et al, Phys. Scr. 76 (2007) 56. 
[10] F. Rahaman et al, gr-qc/0611133v1.

[11] P.K.F. Kuhfittig, Am. J. Phys. 67 (1999) 125.

[12] S.A. Hayward, gr-qc/0306051v1.

[13] M. Jamil, Il Nuovo Cimento B 123 (2008) 599, gr-qc/0806.1319v2.

[14] P.E. Kashargin and S.V. Sushkov, gr-qc/0710.5656v1.

[15] T.K. Dey and S. Sen, gr-qc/0806.4059v1.

[16] N.S. Kardashev et al, Int. J. Mod. Phys. D 16 (2007) 909.

[17] A. Lichnerowicz, Relativistic Hydrodynamics and Magnetohydrodynamics, (W.A. Benjamin, Inc., 1967).

[18] L. Landau and E.M. Lifshitz, The Classical Theory of Fields, (Pergamon Press, 1962).

[19] M. Jamil et al, Eur.Phys.J.C59:907-912,2009, gr-qc/0809.3376v1

[20] S.W. Kim and H. Lee, gr-qc/0102077v1.

[21] P.K.F. Kuhfittig, Class. Quantum Grav. 23 (2006) 5853. 